\title{
Research on Resource Allocation based on RBF Neural Network Under Cloud Computing
}

\author{
Feng Liu ${ }^{1, a^{*}}$, Peiwei Wang ${ }^{2, b}$ and Weijie Zhang ${ }^{3, c}$ \\ ${ }^{1}$ NetWork \& Information Center, Yulin University, Yulin719000, China \\ ${ }^{2}$ College of Information Engineering, Yulin University, Yulin719000, China \\ ${ }^{3}$ Dexin Medical Imageing Technology Co. Ltd.Weinan714000, China \\ aliufeng@yulinu.edu.cn, bwpwkimkibun@163.com, 'zhang.weijie@dexhin.com \\ *The Corresponding author
}

\section{Keywords: Resource allocation; RBF neural network; Cloud computing}

\begin{abstract}
In cloud computing environment, the resources of network is unknown and the topology is not fixed, so the structure and distribution of resources in whole cloud environment and the actual situation is unpredictable. In this case, the position of computing resources and quality amount is unknown to the data section point. It is unknown to find out the computing resources in the network topology by using RBF neural network algorithm, and select the most appropriate one or a few assigned to the user operation until meet the needs of users. This paper studied resource allocation based on RBF neural network under cloud computing. The results show the RBF neural network under cloud computing is a valid method for resource allocation.
\end{abstract}

\section{Introduction}

Due to the rapid development of information technology, when network data gradually complicated, the traditional parallel technology has not required for full foot growing human development and scientific research office [1]. As some network model arose at the historic moment, cloud computing as a new parallel computing technology also appeared on the network[2].As a new Internet, cloud computing is the further development of distributed computing, parallel computing and grid computing based computing model, is also the concept of computer science commercial implementation[3].

Distributed computing tasks will constitute a large number of computer resource pool, so that all applications can root access to computing power, storage space and software services[4]. Cloud computing resources are dynamically scalable and virtualization, through the Internet providing cloud computing is a super computing model based on Internet, and the architecture of a distributed, resource structure of global access, the data center operation in a similar calculation under the environment of Internet are stored in the personal computer, mobile phone and other equipment on the large amount of information and processor resources and work together. Cloud computing services include three levels of service, IaaS, PaaS and SaaS[5].

In recent years, virtualization is the cornerstone of cloud computing, which has also been a hot research field. Virtual tides across all areas of server, storage, network, PC machine etc.. The most prominent advantage of virtualization is to save money, the integrated service server, maximize the utilization of resources.

\section{Resource Allocation Process}

By reference to Map/Reduce computing system, each unit in cloud environment can be divided into two roles: Master and Slave. The former configures NameNode and JobTracker node, the latter configures DataNode and TaskTracker node. When resources are allocated by the main scheduling node of the unit (Master JobTracker) and the units under the jurisdiction of each node in the cluster from the task distribution node (slave TaskTracker) jointly completed. Therefore all nodes in the 
cloud environment unit can be divided into two community structure, master JobTracker master node and all nodes from the slave TaskTracker respectively as a kind of community structure. The master node is responsible for scheduling of all tasks of a job, which is the distribution of data resources of these tasks in different mirror slice of the user, and the user in the mirror slice from the node storage resources, the master node monitoring task execution, re executing the failed task or error handling. The node is responsible for executing the tasks assigned by the master node. From the node after receiving the distribution of the main node, starting from the node to find the appropriate computing node for the storage node of its subordinates. First of all, the amount of their computing resources began to detect from the node, if the rest of the computing resources can meet the users to submit jobs to use, the allocation of computing resources itself, if the remaining resources are not enough to meet the needs of the user to the minimum amount of computing resources, it began to search the cloud computing environment other suitable calculation resources. The RBF neural network will be implemented in this part. The search for in a certain range, the purpose is to prevent the increase of network overhead. If you still can not find a suitable resource scheduling from node to node main operation request to remove user data mirroring the nodes in the cluster partition.

\section{Experiment and Analysis}

Data subsets of four disease images like scar skin, scab are selected for network training, 400 samples are tested, and the corresponding confusion matrix obtained is shown in Fig. 1.

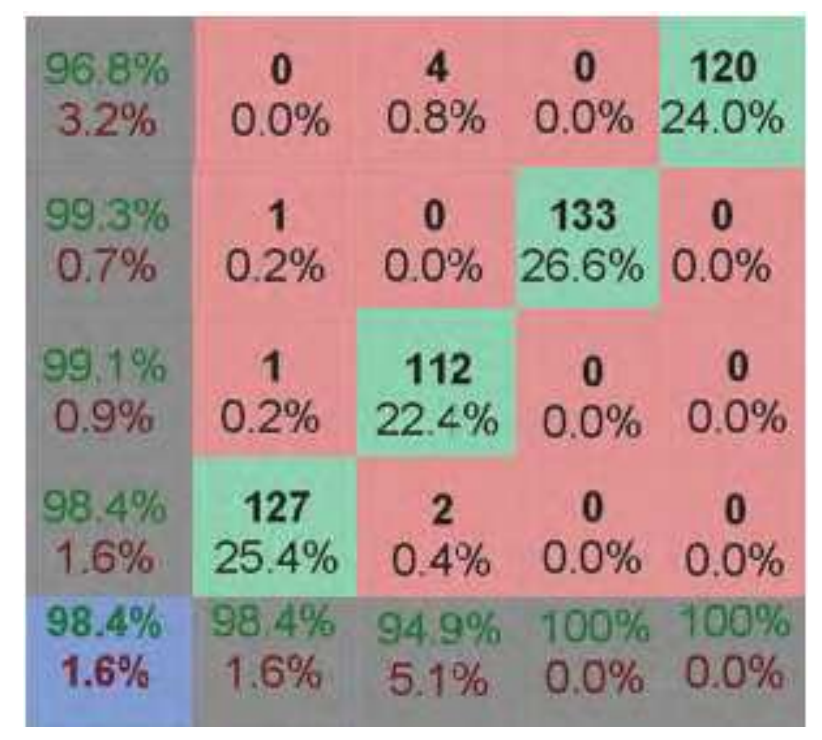

Figure 1. Finite The confusion matrix of target output

Convolution depth study of deep learning neural network selects three momentum algorithms, to observe the iteration round change of loss function of training objectives. Convergence and loss functionis shown in Fig. 2. 


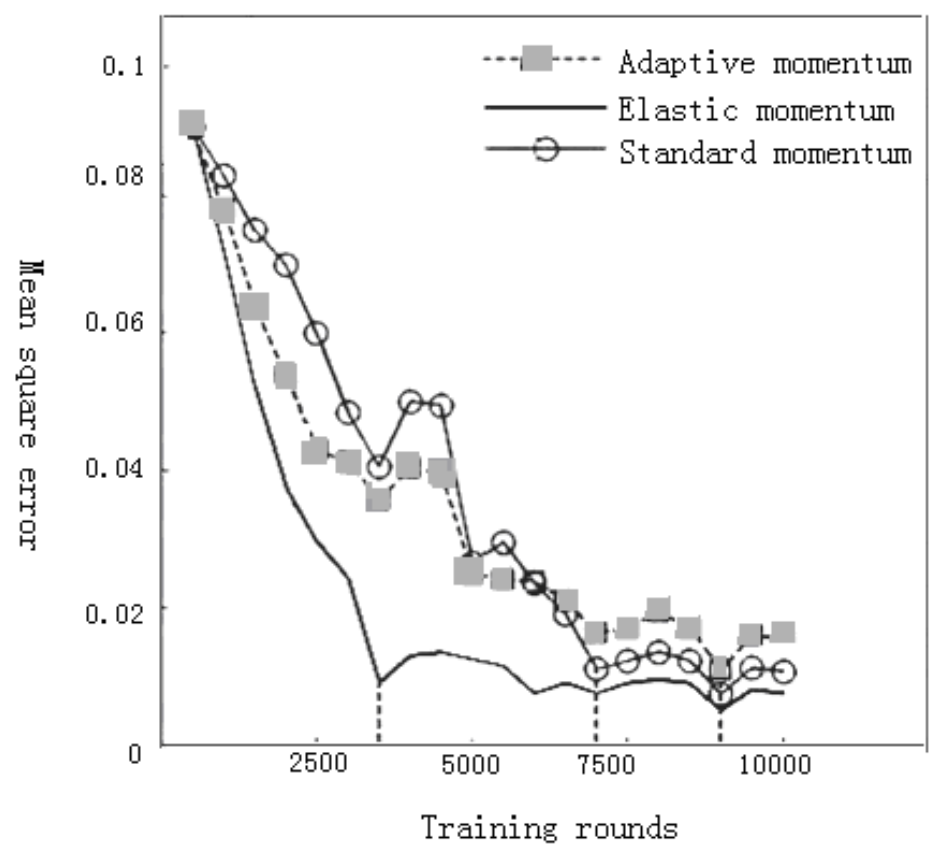

Figure 2. Finite Convergence and loss function

Cloud computing simulation tool Cloud Sim is used to simulate a local area of cloud computing, the purpose is to check the operation of the algorithm in this special grid environment. The software supports the simulation of emerging cloud computing infrastructure and management services.

The modeling and installation of large-scale cloud computing infrastructure, including data can be supported centers on a single physical computing node and a Java virtual machine. Data centers, service agents, scheduling and allocation policies can be modeled. A virtual engine be provided that helps create and manage multiple, independent and collaborative virtual services on a data center node. It is possible to switch between a shared space and a shared time allocation processing core. In the architecture, the Cloud Sim emulator uses a hierarchical structure, bottom-up from SimJava, GridSim, Cloud Sim, user code consists of four levels. The following is the use of the algorithm and the optimal algorithm task completion time, computing power preference task and bandwidth preferences class service comparison chart.
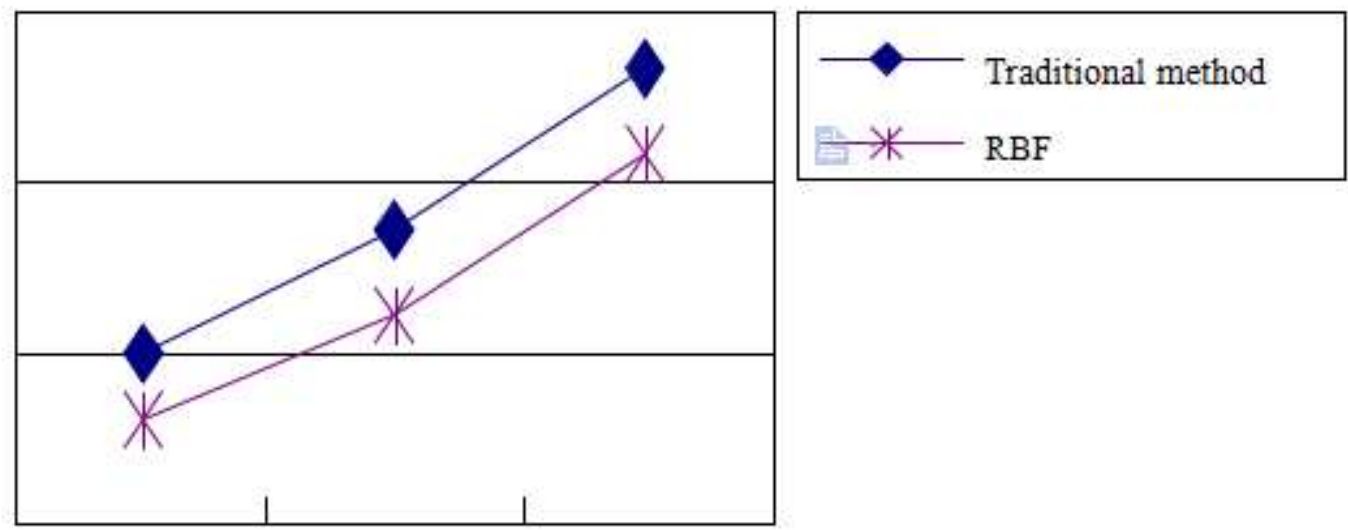

Figure 3. Finite Task completion time comparison chart

Selecting sample sets from Caltech database and NRIA database of California Institute of Technology to convert all the pictures into specification of $28 \times 84$. Accuracy rate, recall ratio and $\mathrm{F}$ value are used as the evaluation indexes. Because of the reciprocal nature and relevance between accuracy rate and recall ratio, low recall ratio brings high accuracy rate, and vice versa. In order to achieve a balance between the two, threshold selection is crucial. Experiments are conducted to 
determine the range of the threshold value, and European distance measure method is applied to observe the curve changes of each section.

This paper studies the pedestrian, and obtains the variation trend diagram among the F value, recall ratio and accuracy rate of pedestrian by taking different threshold values, which is shown below.

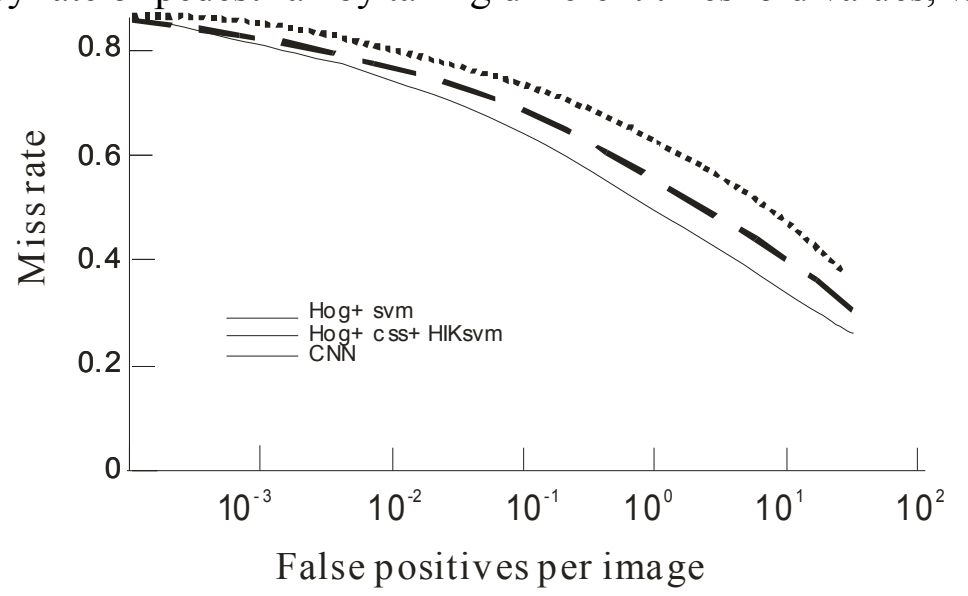

Figure 4. Finite Results Comparison of Algorithms

\section{Summary}

This paper implements QoS task classification and RBF neural network is used to solve the cloud computing resource allocation and scheduling platform based on extended CloudSim source code and compile and re generation, simulation platform and this extension of the algorithm simulation analysis. By comparing the simulation results with the completion time algorithm simulation results show that the proposed optimal, calculation method can effectively perform user tasks, and show better fairness and customer satisfaction.

\section{Acknowledgements}

This work is supported by Key Technological Project on Agricultural Innovation of Shaanxi Science and Technology Department(2016NY-134) and National Natural Science Foundation of China(11641002).

\section{References}

[1] Shyam Sunder Agrawal and Vinod Yadava, Materials and Manufacturing Processes, Vol.19 (2013) No.4, p.122.

[2] P.Asvestas, F. K. Matsopoulos and K.S. Nikita, Journal of Visualization, Vol. 24 (2007) No.7, p.84.

[3] S Luo and B Ren, Comput Methods Programs Biomed, Vol. 34 (2016) No.130, p.154.

[4] Bengio Y, Guyon G and Dror V, Workshop on Unsupervised \& Transfer Learning, Vol.33 (2011) No.7, p.531.

[5] Hinton G E.,Momentum, Vol. 9 (2010) No.1, p.599. 